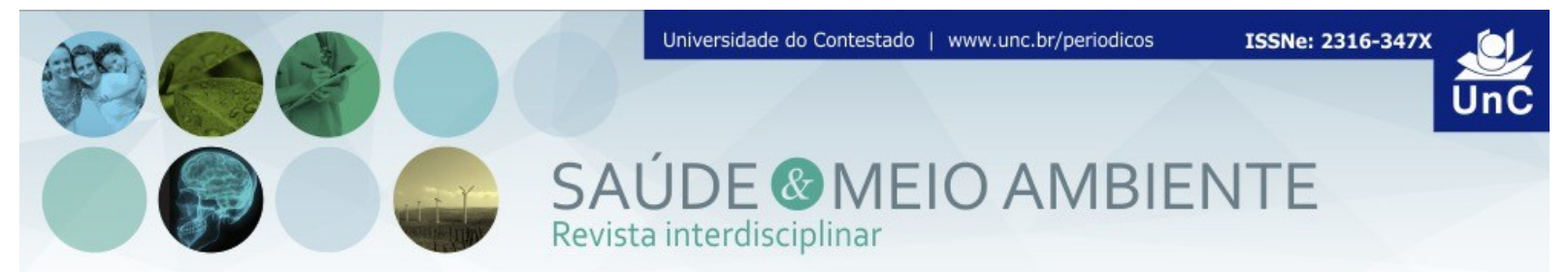

\title{
DIVERSIDADE E ABUNDÂNCIA DA ENTOMOFAUNA EM 3 ESTAÇÕES DO ANO NO CAMPUS DA UNIVERSIDADE DO CONTESTADO - UnC, DISTRITO DE MARCÍLIO DIAS, SANTA CATARINA
}

RESUMO: O objetivo do presente estudo foi avaliar a estrutura da comunidade entomológica no Campus da Universidade do Contestado, distrito de Marcílio Dias em 3 estações do ano. Para tanto, foram utilizadas três armadilhas de interceptação de vôo, tipo Malaise. Foi coletado um total de 53.622 indivíduos, distribuídos em 21 ordens no período de fevereiro a setembro de 2014. A armadilha localizada no ponto 3 obteve as maiores frequências de captura $(37,96 \%)$, seguida da armadilha 2 $(31,07 \%)$ e $1(30,97 \%)$. A estação de inverno foi a mais representativa em termos de número de indivíduos para as 3 armadilhas $(n=23836)$, seguida de outono $(n=15690)$ e verão ( $n=14096)$. No entanto, o maior número de indivíduos coletados no inverno é atribuído apenas a 3 ordens: Diptera, Collembola e Acari. As ordens de insetos que apresentaram constância em todos os meses de estudo foram Coleoptera e Diptera, sendo que esta última ordem apresentou também as maiores frequências nos três pontos de amostragem, com 66,12\%, 42,76\% e 62.5\% nas armadilhas Malaise 1, 2 e 3, respectivamente. Estudos que foquem levantamentos da entomofauna na região são de suma importância, uma vez que há notável carência de trabalhos de cunho semelhante, inviabilizando, inclusive, a realização de maiores comparações através de bibliografias. Por este motivo, torna-se imprescindível um esforço maior no conhecimento da prevalência destes insetos, que muitas vezes são ignorados, apesar da importância que exercem na manutenção do equilíbrio ecológico.

Palavras-chave: Entomofauna. Diversidade. Abundância. Malaise.

\footnotetext{
${ }^{1}$ Bióloga, Me. CRBio 69392-03D. Departamento de Engenharia Florestal, Laboratório de Entomologia. Universidade do Contestado. Santa Catarina. Brasil. E-mail: bordin.daliana@gmail.com

${ }^{2}$ Mestre em Ciências Ambientais pela Universidade Comunitária da Região de Chapecó, Brasil (2010). Professor do Curso de Engenharia Florestal, Laboratório de Entomologia. Universidade do Contestado. Santa Catarina. Brasil. E-mail: vitor@unc.br
} 


\title{
DIVERSITY AND ABUNDANCE IN ENTOMOFAUNA 3 SEASONS IN THE CAMPUS OF THE UNIVERSITY CONTESTED - UnC, MARCILIO DIAS DISTRICT, SANTA CATARINA
}

\begin{abstract}
The aim of this study was to evaluate the structure of entomological community in the Universidade do Contestado campus, Marcilio Dias district, during three seasons of the year. For this, we used three flight intercept traps, type Malaise. A total of 53,622 individuals belonging to 21 orders in the period from February to September 2014 was collected. The trap located at point 3 had the highest capture frequency $(37.96 \%)$, followed by trap $2(31.07 \%)$ and trap $1(30.97 \%)$. The winter season was the most representative in terms of number of subjects for the 3 traps (n $=23836)$, followed by the fall $(n=15690)$ and summer $(n=14096)$. However, the largest number of individuals collected in winter is assigned only 3 orders: Diptera, Collembola and Acari. The orders of insects that showed consistency in all the months of study were Coleoptera and Diptera, and the latter order also had the highest frequency in the three sampling points, with $66.12 \%, 42.76 \%$ and $62.5 \%$ in Malaise traps 1, 2 and 3, respectively. Studies that focus on surveys of insect fauna in the region are very importance, since there is notable lack of similar nature of work, making it impossible even to enhanced comparisons through bibliographies. For this reason, it is essential efforts for greater knowledge about the prevalence of these insects, which are often ignored, despite the importance of their role in maintaining the ecological balance.
\end{abstract}

Keywords: Entomofauna. Diversity. Abundance. Malaise.

\section{INTRODUÇÃO}

De acordo com Brown e Freitas (2002), a composição típica da fauna de insetos em cada região parece responder diferencialmente à área, distúrbios e vários fatores micro-ambientais, em parte, devido às diversas espécies comuns ou dominantes e à presença de distintos recursos das plantas.

Conforme Copatti e Gasparetto (2012), insetos geralmente apresentam elevadas densidades populacionais e diversidade, além de grande variedade de respostas à qualidade e à quantidade de recursos disponíveis, desempenhando importante papel no funcionamento dos ecossistemas, atuando como predadores, parasitas, detritívoros, polinizadores, entre outros, dentro de complexas redes tróficas.

Pesquisas que contemplam levantamentos populacionais são importantes instrumentos para a avaliação da biodiversidade, pois evidenciam a densidade populacional das diferentes espécies e permitem caracterizar as comunidades estudadas por meio dos índices faunísticos. E, quando se tratam da fauna entomológica, podem estabelecer a potencialidade de danos de insetos às culturas florestais e agrícolas e auxiliar na utilização de métodos de controle dos mesmos. 
Para Couceiro e colaboradores, 2002, estudos sobre entomofauna em ecossistemas são importantes, pois os insetos fazem parte das cadeias tróficas, ciclagem de nutrientes e podem também ser utilizados como bioindicadores. Grande parte da entomofauna ainda é desconhecida e, geralmente, estudos sobre ambientes alterados não possuem um banco de informações anteriores à perturbação.

Alguns insetos são considerados "espécies-chave" porque a perda de suas funções ecológicas críticas poderia levar um ecossistema inteiro ao colapso. Alguns insetos estão intimamente associados às dificuldades da existência humana, uma vez que certos insetos causam danos à saúde e outros afetam de forma negativa atividades de agricultura e horticultura. Por outro lado, há insetos que trazem muitos benefícios à sociedade humana, tanto por nos fornecer alimento diretamente quanto por contribuir para a produção de determinados materiais de consumo (GALLO et al., 2002).

Estudos que foquem levantamentos da entomofauna na região são de suma importância, uma vez que há notável carência de trabalhos de cunho semelhante, inviabilizando, inclusive, a realização de maiores comparações através de bibliografias. Muitos dos estudos realizados em Santa Catarina têm foco nas associações de insetos a algum gênero botânico (DORNELES, 2010; GONÇALVES, 1997) ou, muitas vezes, não contemplam várias ordens de insetos, mas tratam de um táxon em específico (PERIN, 2009; WOLDAM, 2007).

Por este motivo, torna-se imprescindível um esforço maior no conhecimento da prevalência destes insetos, que muitas vezes são ignorados, apesar da importância que exercem na manutenção do equilíbrio ecológico.

As armadilhas Malaise, artefato de captura do presente estudo, são comumente empregadas como ferramentas de coleta da fauna entomológica em vários aspectos e tem se mostrado um método eficiente para mostrar a abundância relativa (CUOZZO et al., 2011; OLIVEIRA et al., 2006), a diversidade (OLIVEIRA et al., 2013), a sazonalidade (OLIVEIRA et al., 2006), a estabilidade da entomofauna (LARANJEIRO, 2003) e a avaliação de bioindicadores em agrosistemas (PEREIRA, 2009).

Embora demonstre seletividade para a captura das ordens Hymenoptera, Diptera e Lepidoptera (HUSCH et al., 2010; LARANJEIRO, 2003; MARINONI; BONATTO, 2002), este tipo de ferramenta é utilizada para representar várias outras ordens de insetos isoladamente, como Neuroptera (VAS et al., 2001), parasitóides da ordem Hymenoptera (MARCHIORI et al., 2001) e famílias da ordem coleoptera (WOLDAM, 2007; OLIVEIRA, 2006; MARINONI; DUTRA, 1997), por exemplo.

Diante do exposto e das poucas informações existentes sobre os insetos, no município de Canoinhas - SC, e, considerando a importância ecológica deste grupo, o presente trabalho teve como objetivo avaliar a estrutura da comunidade entomológica no Campus da Universidade do Contestado - UNC, distrito de Marcílio Dias, em 3 estações do ano (verão, outono, inverno) em três pontos distintos, através de armadilhas do tipo Malaise. 
Desta forma, o presente estudo visa aprimorar e divulgar o conhecimento a respeito da entomofauna local, como subsídio para atividades locais de manejo e conservação. Esses tipos de dados podem levar a conhecimentos imprescindíveis para a avaliação e preservação da biodiversidade das espécies raras ou endêmicas nos remanescentes dos ecossistemas naturais.

\section{MATERIAIS E MÉTODOS}

O presente estudo foi executado através de pesquisa de campo, pesquisa laboratorial e pesquisas bibliográficas tendo embasamento tanto em aspectos qualitativos quanto em aspectos quantitativos. Foi realizado no Campus da Universidade do Contestado - UnC, distrito de Marcílio Dias no período de janeiro de 2014 a setembro de 2014, em três pontos específicos.

O Campus está localizado na Bacia Hidrográfica do Rio Canoinhas, no Planalto Norte Catarinense, entre a latitude $26^{\circ} 05^{\prime} \mathrm{S}$ e a longitude $50^{\circ} 23^{\prime} \mathrm{W}$, no município de Canoinhas, SC e é margeado por fragmentos de Floresta Ombrófila Mista em estágio secundário.

De acordo com Copatti e Gasparetto (2012), além da araucária, as principais espécies arbóreas encontradas na floresta ombrófila mista são: caroba (Jacaranda micrantha), angico-vermelho (Parapiptadenia rigida), canela (Ocotea sp.), camboatá (Matayba elaeagnoides), guajuvira (Patogonula americana), cabreúva-parda (Myrocarpus frondosus), cedro (Cedrela fissilis), coqueiro-jerivá (Syagrus romanzoffiana), ipê amarelo (Tabebuia alba), pitangueira (Eugenia uniflora), branquilho (Sebastiana commersoniana) e urtigão (Urera baccifera).

\section{OBTENÇÃO DOS EXEMPLARES}

Para o levantamento da entomofauna foram utilizadas três armadilhas de interceptação de vôo, tipo Malaise (TOWNES, 1972) (Figura 1).

As armadilhas foram instaladas em três pontos distintos (Figura 2). O primeiro ponto localiza-se nas coordenadas $26^{\circ} 5^{\prime} 28.49^{\prime \prime S}$ e $50^{\circ} 23^{\prime} 16.25^{\prime \prime} \mathrm{O}$, ao lado da trilha ecológica da instituição. O segundo ponto, $26^{\circ} 5^{\prime} 30.68^{\prime \prime S}$ e $50^{\circ} 23^{\prime} 17.37$ "O, refere-se a uma área aberta, local de influência da comunidade acadêmica. O terceiro ponto está inserido em uma área de mata mais fechada, localizada em $26^{\circ} 5^{\prime} 30.68^{\prime \prime S ~ e ~}$ $50^{\circ} 23$ '21.05”O.

As armadilhas foram montadas dia 29 de Janeiro de 2014 e retirados os indivíduos semanalmente até dia 25 de setembro de 2014, período que compreendeu 3 estações do ano. 
Figura 1- Uma das três armadilhas Malaise instaladas.

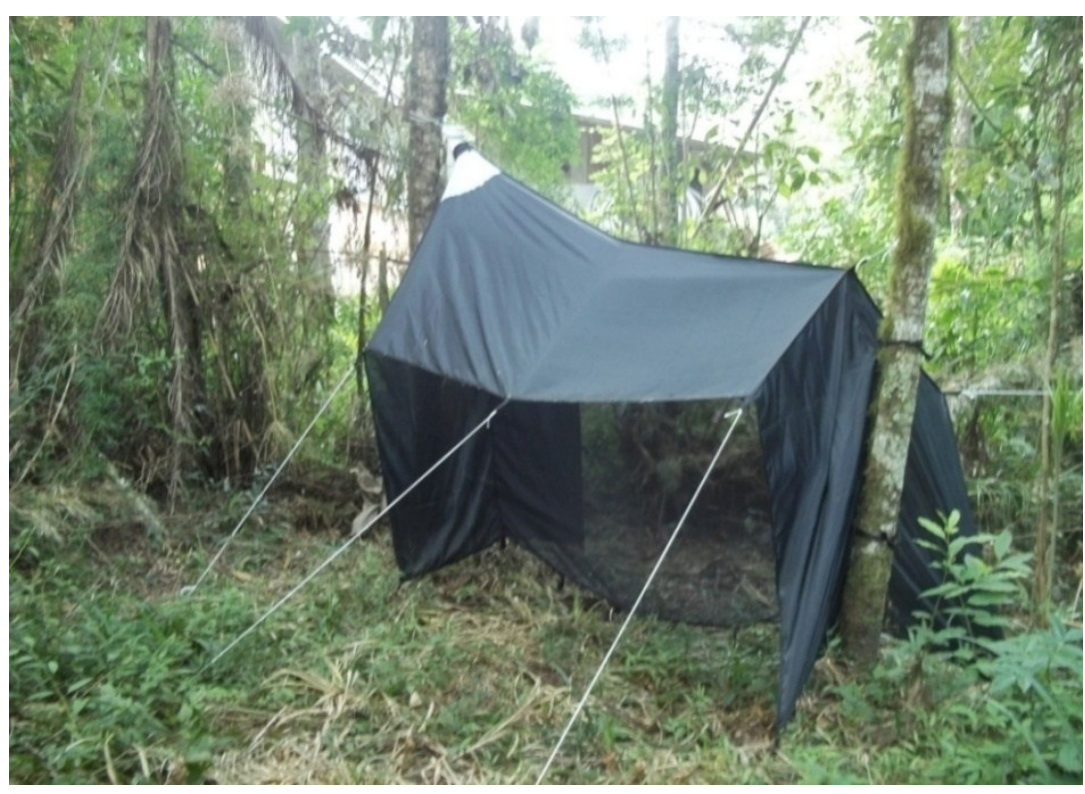

Fonte: Dados da pesquisa (2015).

Para a padronização das estações do ano, os meses foram agrupados em fevereiro e março (verão); abril, maio junho (outono), julho, agosto e setembro (inverno).

Figura 2- Locais de amostragem da entomofauna no Campus da UNC - Marcílio Dias.

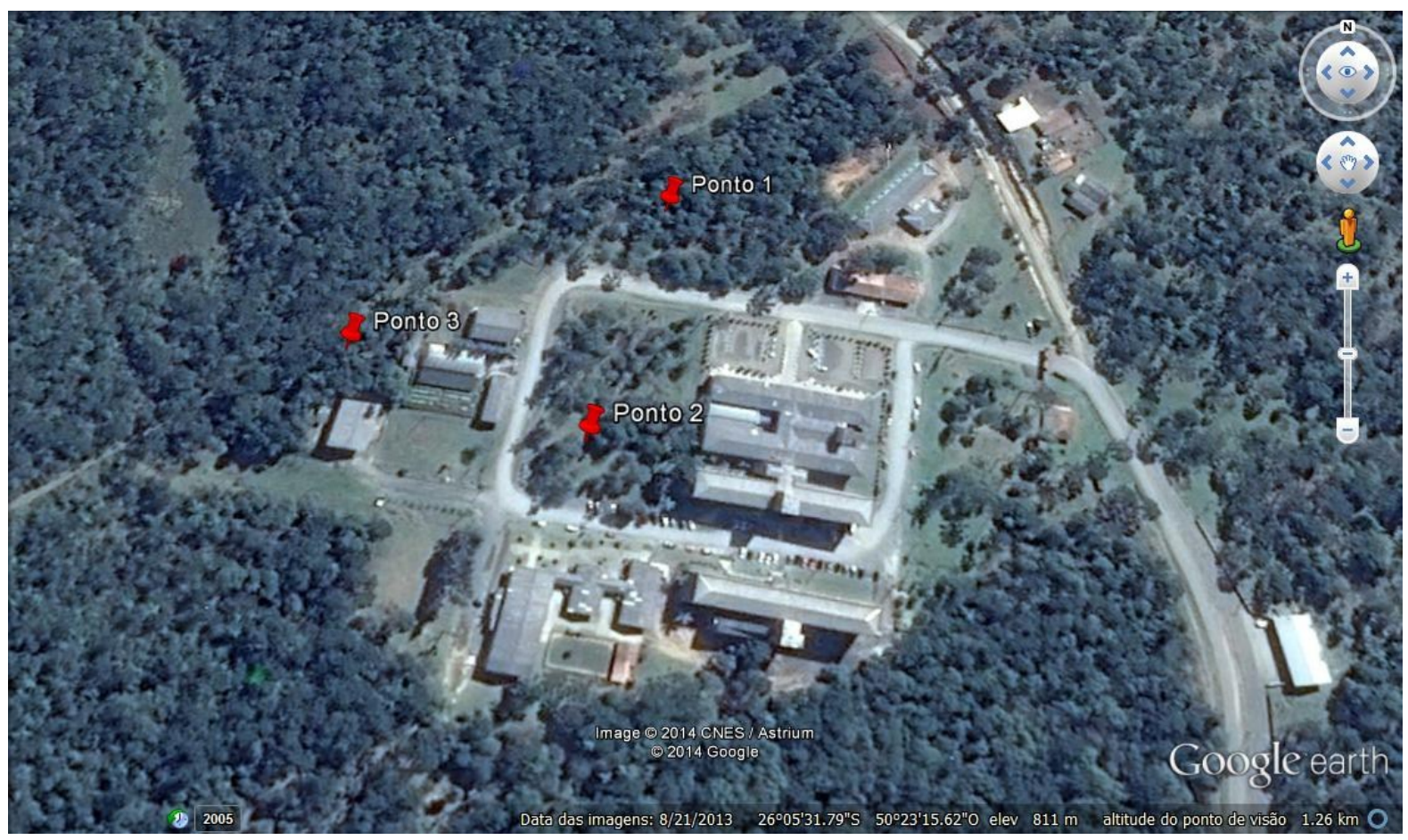

Fonte: Google Earth

Saúde Meio Ambient. v. 5, n. 1, p. 89-104, jan./jun. 2016 


\section{ARMAZENAMENTO E IDENTIFICAÇÃO DOS EXEMPLARES}

O material coletado foi armazenado em frascos devidamente identificados e fixado com solução de álcool $70 \%$. Os indivíduos foram triados e identificados em nível de ordem em microscópio estereoscópio e com o auxílio de guias especializados.

Após determinação de toda a entomofauna coletada, os indivíduos foram etiquetados com os dados de coleta e identificação taxonômica, tombados e depositados no Laboratório de Entomologia da Universidade do Contestado - UNC para serem utilizados como coleção de referência e realização de estudos posteriores.

\section{TRATAMENTO DOS DADOS}

A frequência (f) das ordens foi determinada pela participação percentual do número de indivíduos de cada ordem, em relação ao total coletado, de acordo com a fórmula: $\mathrm{f}=(\mathrm{ni} / \mathrm{N}) \times 100$, em que: $\mathrm{f}=$ frequência relativa; $\mathrm{ni}$ = número total de indivíduos da ordem i; $\mathrm{N}$ = número total de indivíduos (SILVEIRA-NETO et al., 1976).

A dominância das ordens foi definida de acordo com as categorias estabelecidas por Friebe (1983), a partir da abundância relativa de cada espécie, segundo a fórmula: $\mathrm{D} \%=(\mathrm{i} / \mathrm{t}) \times 100$ em que: $\mathrm{D} \%=$ porcentagem de dominância; $i=$ número total de indivíduos de uma ordem; $\mathrm{t}=$ total de indivíduos coletados. Sendo as ordens consideradas como: Eudominante > 10\%; Dominante $>5-10 \%$; Subdominante $>2-5 \%$; Recessiva $=1-2 \%$ e Rara $<1 \%$.

A constância foi calculada por meio da porcentagem de ocorrência das ordens no levantamento, utilizando-se da fórmula: $\mathrm{C}=(\mathrm{ci} / \mathrm{Nc}) \times 100$, em que: $\mathrm{C}=$ percentagem de constância; $\mathrm{ci}=$ número de coletas contendo a ordem $\mathrm{i} ; \mathrm{Nc}=$ número total de coletas efetuadas. Pelas percentagens, as ordens foram agrupadas em categorias, segundo Silveira-Neto et al. (1976): Ordens constantes (x): presentes em mais de $50 \%$ das coletas; Ordens acessórias (y): presentes entre 25 e $50 \%$ das coletas; Ordens acidentais (z): presentes em menos de $25 \%$ das coletas.

\section{RESULTADOS E DISCUSSÃO}

Foi coletado um total de 53.622 indivíduos, distribuídos em 21 ordens no período de fevereiro a setembro de 2014. Estes números foram semelhantes ou superiores ao encontrado em outros estudos com o mesmo artefato de captura 
(OLIVEIRA et al., 2013; HUSCH et al., 2010; OLIVEIRA et al., 2006; DUTRA; MARINONI, 1994).

A armadilha localizada no ponto 3 obteve as maiores frequências de captura $(37,96 \%)$, seguida da armadilha $2(31,07 \%)$ e $1(30,97 \%)$ (Gráfico 1).

Em termos de número de ordens por armadilha, a Malaise 3 foi a mais diversa, com 21 ordens, sendo 18 delas atribuídas a fauna entomológica. As armadilhas 1 e 2 apresentaram 19 ordens, sendo 16 destas representadas pelos insetos. Três ordens referem-se a fauna acompanhante, Acari, Arachnida e Pulmonata e foram consideradas no presente estudo pois partilham recursos e hábitat com as demais ordens coletadas. Muitos estudos que utilizam Malaise como ferramenta de coleta não consideram as outras ordens ocorrentes (OLIVEIRA et al., 2006; DUTRA; MARINONI, 1994), pois acreditam que este tipo de armadilha é seletiva para a entomofauna. No entanto, alguns trabalhos demonstram que estes artefatos são também adequados para o estudo de outros indivíduos, e que o aparecimento de outras ordens é bem mais do que ocasional, como exemplificado para os aracnídeos (JENNINGS; HILBURN, 1998).

Gráfico 1 - Frequências (\%) de captura para as três armadilhas.

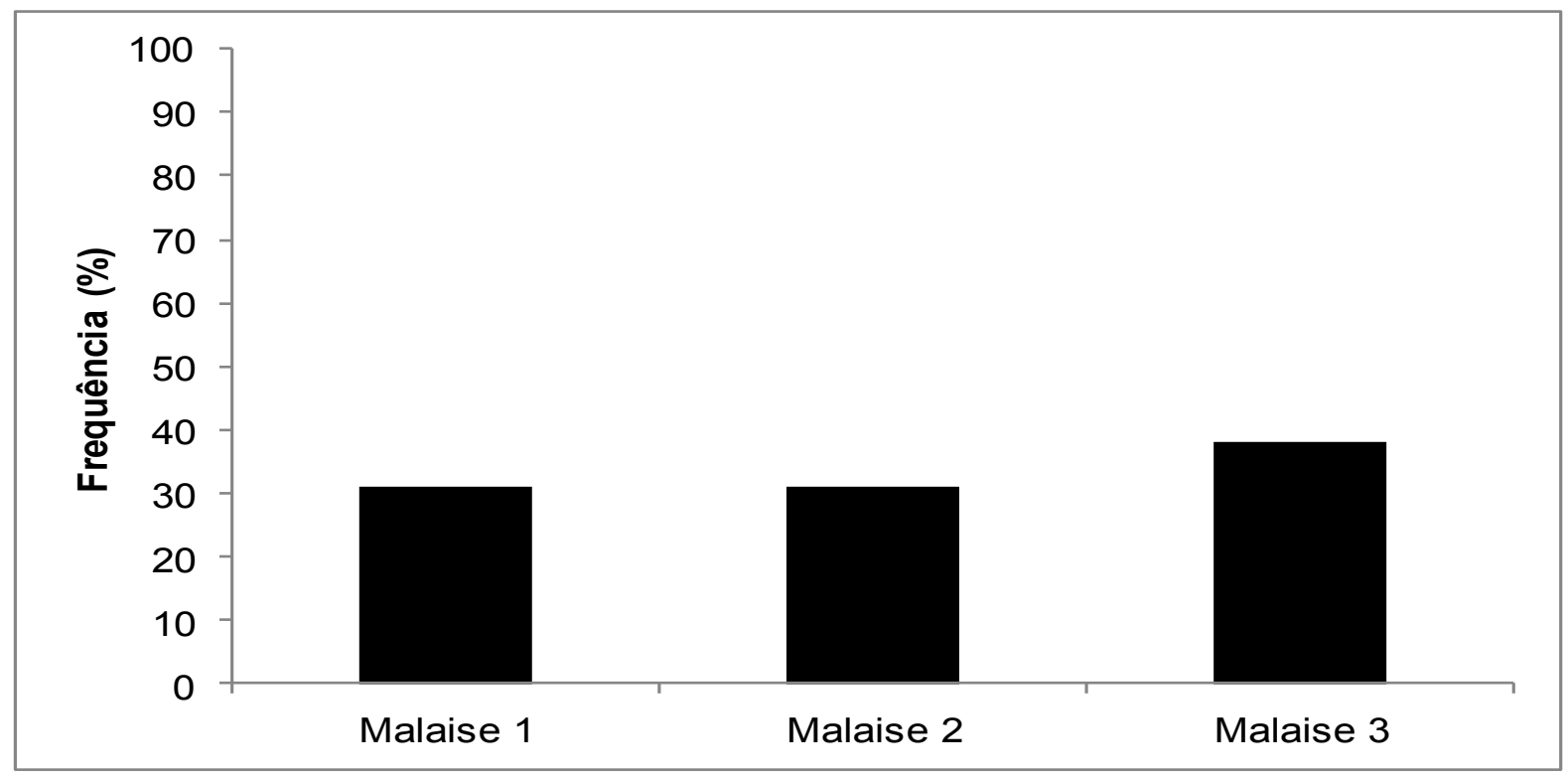

Fonte: Dados da pesquisa (2015).

COMPARAÇÃO ENTRE AS TRÊS ARMADILHAS

A armadilha 1 foi responsável pela captura de 16608 indivíduos, sendo que 10962 destes foram representados somente pela ordem Diptera (Gráfico 2). 
Gráfico 2 - Frequência (\%) de indivíduos coletados na armadilha Malaise 1 distribuída por ordem.

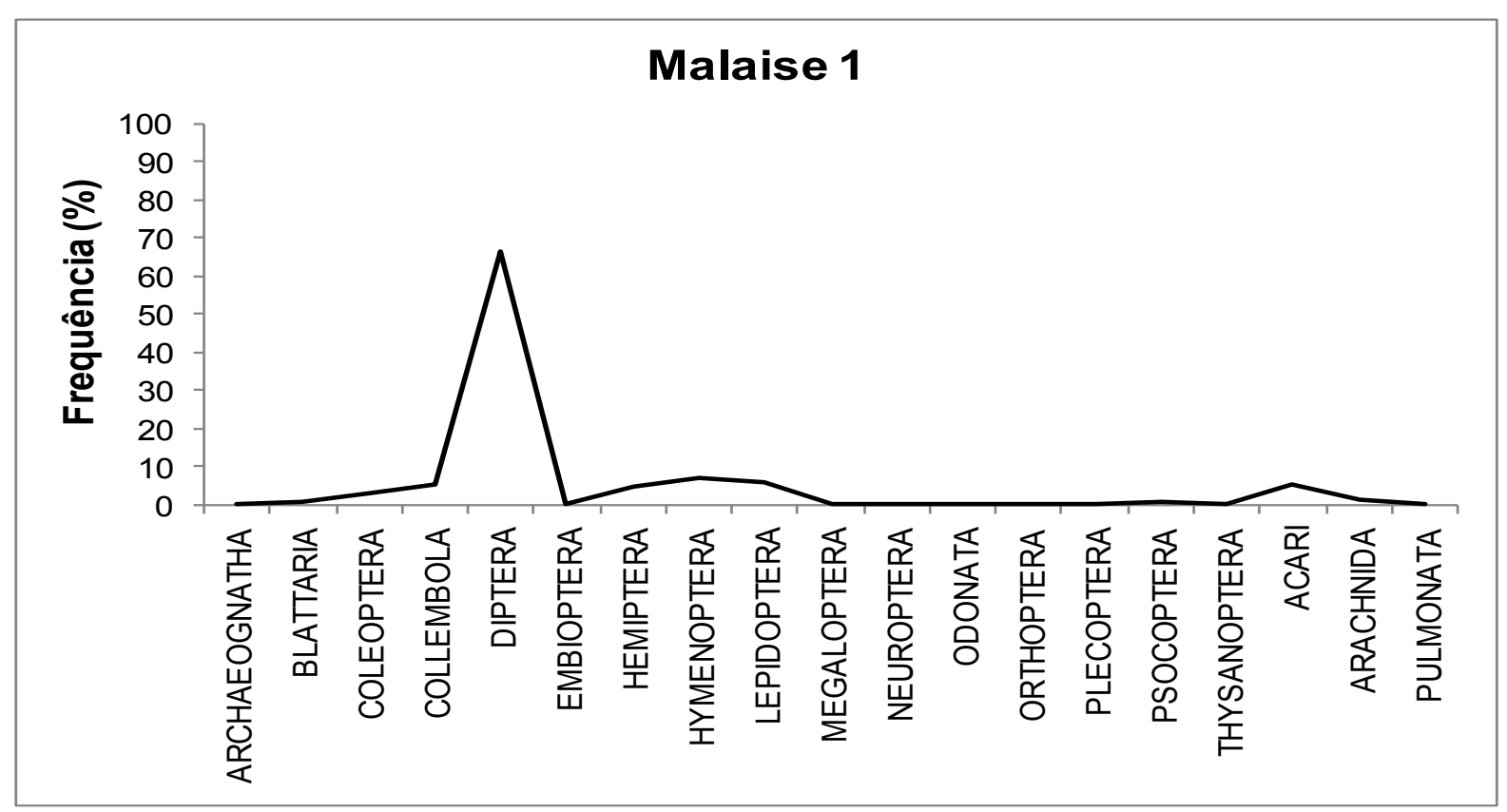

Fonte: Dados da pesquisa (2015).

As armadilhas 2 e 3 apresentaram 16658 e 20356 indivíduos, respectivamente, sendo que a maior representatividade em termos de captura também se deu pela ordem Diptera (Gráficos 3 e 4).

A ordem Diptera é citada em muitos estudos como o grupo mais abundante (OLIVEIRA et al., 2013; HUSCH et al., 2010; OLIVEIRA et al., 2006; MARINONI; BONATTO, 2002; DUTRA; MARINONI, 1994) e que praticamente determinam as flutuações observadas para o conjunto dos Insecta.

Gráfico 3 - Frequência (\%) de indivíduos coletados na armadilha Malaise 2 distribuída por ordem.

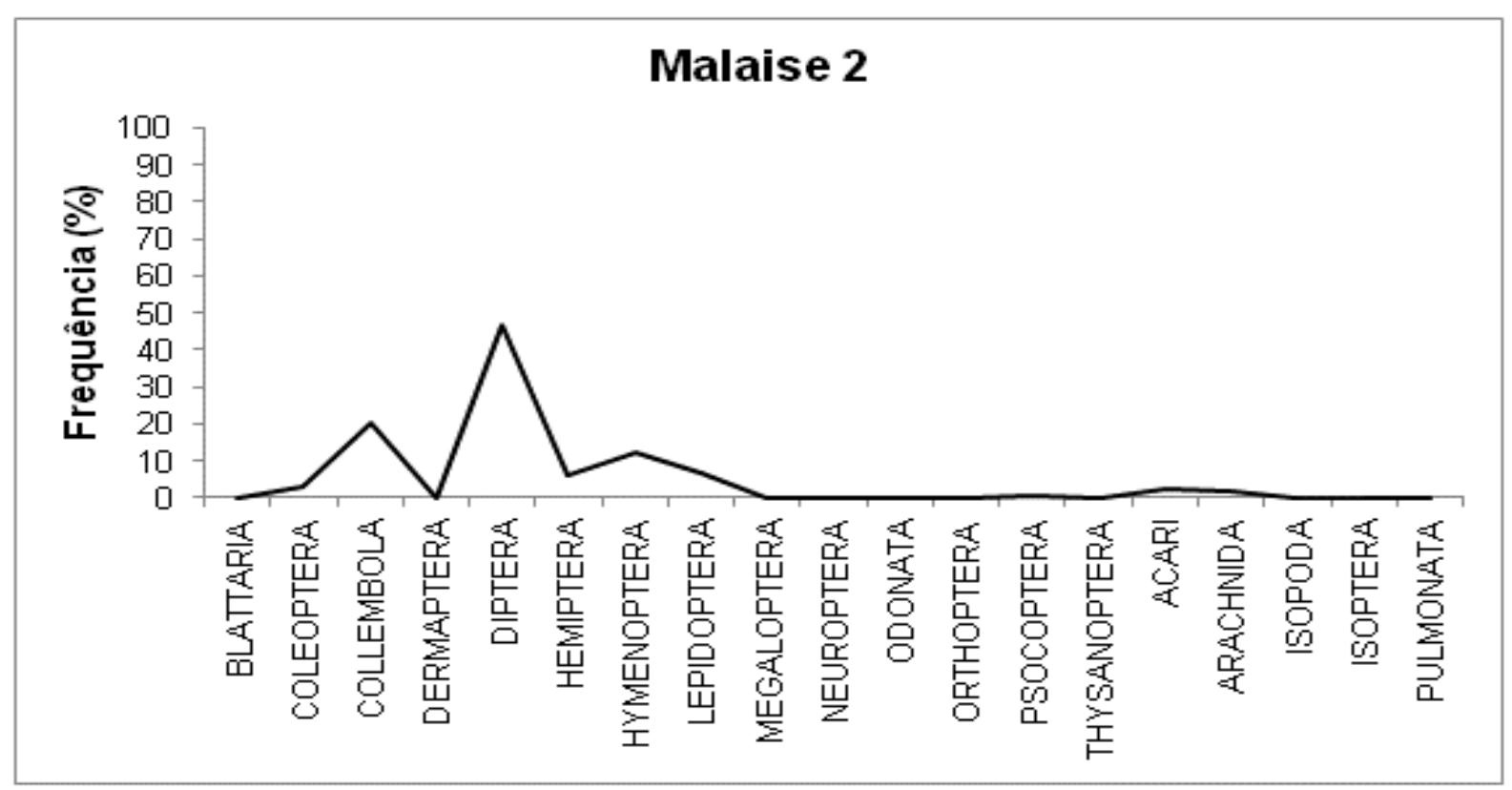

Fonte: Dados da pesquisa (2015). 
Gráfico 4 - Frequência (\%) de indivíduos coletados na armadilha Malaise 3 distribuída por ordem.

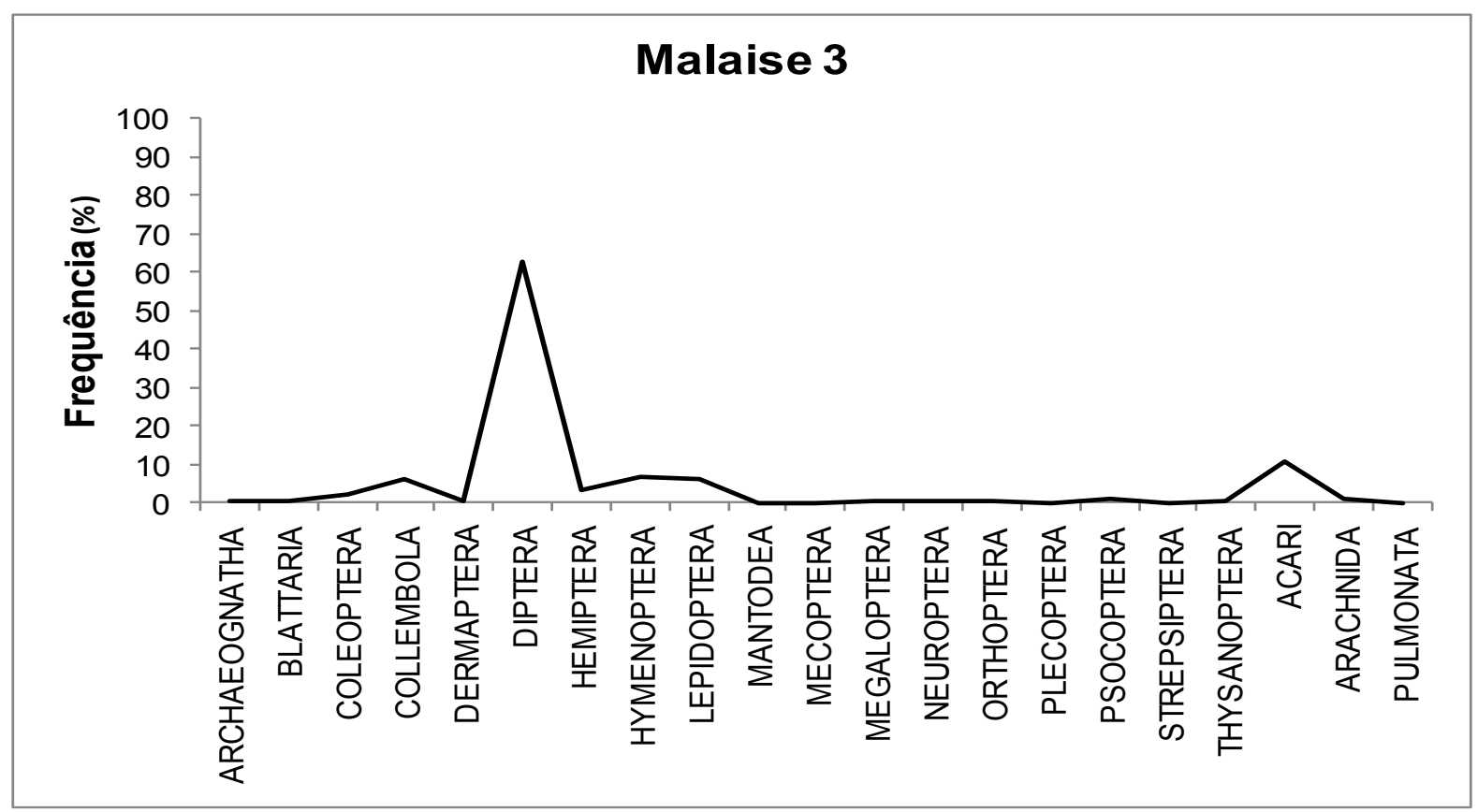

Fonte: Dados da pesquisa (2015).

\section{DIVERSIDADE E ABUNDÂNCIA DE INSETOS ENTRE AS ESTAÇÕES DO ANO}

Não houve padrão no número e diversidade de insetos coletados entre as armadilhas ao longo dos meses de estudo. No entanto, as maiores capturas ocorreram em setembro, para as três armadilhas e as menores capturas, em agosto (Malaise 1), junho (Malaise 2) e maio (Malaise 3).

De acordo com a sazonalidade, a estação de inverno foi a mais representativa em termos de número de indivíduos para as 3 armadilhas $(n=23836)$, seguida de outono $(n=15690)$ e verão $(n=14096)$ (Gráfico 5). No entanto, o maior número de indivíduos coletados no inverno é atribuído apenas a 3 ordens: Diptera, Collembola e Acari.

A queda de temperatura característica da estação de inverno pode ser um fator limitante para o aparecimento de algumas ordens, sendo as maiores capturas desta estação relacionadas às ordens eudominantes e dominantes, provavelmente devido às suas características generalistas e adaptabilidade às variadas condições ambientais.

Husch e colaboradores, (2010) atribuem a diminuição na abundância de insetos nos meses mais quentes à quantidade de chuvas e umidade relativa, típico dos meses fevereiro e março.

O outono apresentou suas maiores capturas atribuídas à Diptera, Collembola, Lepidoptera e Hymenoptera e o verão à Diptera, Hymenoptera e Lepidoptera. 
Os resultados obtidos concordam com as observações de muitos autores quanto à capacidade seletiva da armadilha Malaise, notadamente para Diptera, Hymenoptera e Lepidoptera (OLIVEIRA et al., 2013; DUTRA; MARINONI, 1994).

Gráfico 5 - Número de indivíduos coletados nas três estações do ano em cada armadilha.

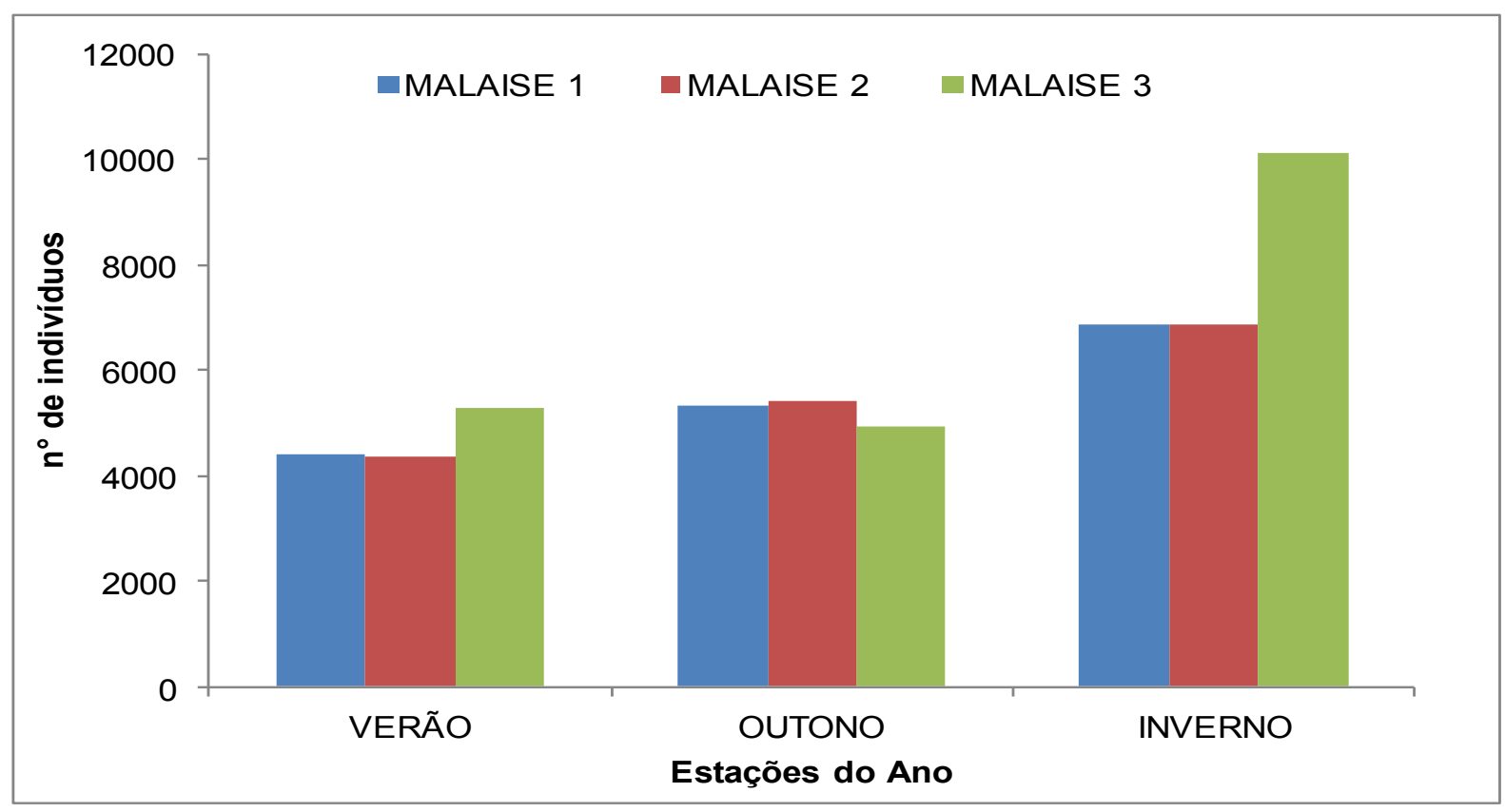

Fonte: Dados da pesquisa (2015).

FREQUÊNCIA E CONSTÂNCIA DOS EXEMPLARES OBTIDOS

De acordo com os cálculos de frequência e constância pôde-se ter noção da utilização do hábitat pelas diferentes ordens, sendo que a maioria delas foi classificada como rara e constante, como observado nas Tabelas 1, 2 e 3.

As ordens de insetos que apresentaram constância em todos os meses de estudo foram Coleoptera e Diptera, sendo que esta última ordem apresentou também as maiores freqüências nos três pontos de amostragem, com $66,12 \%$, $42,76 \%$ e $62.5 \%$ nas armadilhas Malaise 1, 2 e 3, respectivamente. Estes resultados corroboram com o estudo de Husch et al. (2010), que classificou Diptera como constante nos oito pontos de coleta, aparecendo em mais de $50 \%$ dos levantamentos efetuados e apresentando maior frequência em cinco dos seis locais amostrados, correspondendo a mais de $68 \%$ do total de insetos coletados. 
Tabela 1 - Número de indivíduos (N), frequência (\%), constância e dominância (\%) de ordens coletadas com a armadilha Malaise 1 no período de fevereiro de 2014 a setembro de 2014.

\begin{tabular}{|c|c|c|c|c|c|}
\hline \multirow[b]{2}{*}{ ORDEM } & \multicolumn{4}{|c|}{ Malaise 1} & \multirow[b]{2}{*}{ Classificação } \\
\hline & $\mathbf{n}$ & Frequência (\%) & Classificação & Constância (C) & \\
\hline ARCHAEOGNATHA & 16 & 0,10 & Rara & 87,50 & Constante \\
\hline BLATTARIA & 66 & 0,40 & Rara & 100,00 & Constante \\
\hline COLEOPTERA & 518 & 3,12 & Subdominante & 100,00 & Constante \\
\hline COLLEMBOLA & 894 & 5,38 & Dominante & 87,50 & Constante \\
\hline DERMAPTERA & - & - & - & - & - \\
\hline DIPTERA & 10982 & 66,12 & Eudominante & 100,00 & Constante \\
\hline EMBIOPTERA & 4 & 0,02 & Rara & 12,50 & Acidental \\
\hline HEMIPTERA & 768 & 4,62 & Subdominante & 87,50 & Constante \\
\hline HYMENOPTERA & 1127 & 6,79 & Dominante & 87,50 & Constante \\
\hline LEPIDOPTERA & 916 & 5,52 & Dominante & 87,50 & Constante \\
\hline MANTODEA & - & - & - & - & - \\
\hline MECOPTERA & - & - & - & - & - \\
\hline MEGALOPTERA & 11 & 0,07 & Rara & 75,00 & Constante \\
\hline NEUROPTERA & 4 & 0,02 & Rara & 25,00 & Acessória \\
\hline ODONATA & 1 & 0,01 & Rara & 12,50 & Acidental \\
\hline ORTHOPTERA & 27 & 0,16 & Rara & 87,50 & Constante \\
\hline PLECOPTERA & 6 & 0,04 & Rara & 12,50 & Acidental \\
\hline PSOCOPTERA & 121 & 0,73 & Rara & 100,00 & Constante \\
\hline STREPSIPTERA & - & - & - & - & - \\
\hline THYSANOPTERA & 8 & 0,05 & Rara & 50,0 & Acessória \\
\hline ACARI & 899 & 5,41 & Dominante & 62,50 & Constante \\
\hline ARACHNIDA & 239 & 1,44 & Recessiva & 100,00 & Constante \\
\hline ISOPODA & - & - & - & - & - \\
\hline ISOPTERA & - & - & - & - & - \\
\hline PULMONATA & 1 & 0,01 & Rara & 12,50 & Acidental \\
\hline TOTAL & 16608 & 100,00 & & & \\
\hline
\end{tabular}

Fonte: Dados da pesquisa (2015). 
Tabela 2 - Número de indivíduos (N), frequência (\%), constância e dominância (\%) de ordens coletadas com a armadilha Malaise 2 no período de fevereiro de 2014 a setembro de 2014.

\begin{tabular}{|c|c|c|c|c|c|}
\hline \multicolumn{6}{|c|}{ Malaise 2} \\
\hline ORDEM & $\mathbf{n}$ & Frequência (\%) & Classificação & Constância (C) & Classificação \\
\hline ARCHAEOGNATHA & - & - & - & - & - \\
\hline BLATTARIA & 16 & 0,10 & Rara & 62,5 & Constante \\
\hline COLEOPTERA & 490 & 2,94 & Subdominante & 100 & Constante \\
\hline COLLEMBOLA & 3406 & 20,45 & Eudominante & 100 & Constante \\
\hline DERMAPTERA & 2 & 0,01 & Rara & 12,5 & Acidental \\
\hline DIPTERA & 7789 & 46,76 & Eudominante & 100 & Constante \\
\hline EMBIOPTERA & - & - & - & - & - \\
\hline HEMIPTERA & 1041 & 6,25 & Dominante & 100 & Constante \\
\hline HYMENOPTERA & 2049 & 12,30 & Eudominante & 100 & Constante \\
\hline LEPIDOPTERA & 1104 & 6,63 & Dominante & 100 & Constante \\
\hline MANTODEA & - & - & - & - & - \\
\hline MECOPTERA & - & - & - & - & - \\
\hline MEGALOPTERA & 2 & 0,01 & Rara & 25 & Acessória \\
\hline NEUROPTERA & 2 & 0,01 & Rara & 25 & Acessória \\
\hline ODONATA & 1 & 0,01 & Rara & 12,5 & Acidental \\
\hline ORTHOPTERA & 18 & 0,11 & Rara & 62,5 & Constante \\
\hline PLECOPTERA & - & - & - & - & - \\
\hline PSOCOPTERA & 121 & 0,73 & Rara & 87,5 & Constante \\
\hline STREPSIPTERA & - & - & - & - & - \\
\hline THYSANOPTERA & 7 & 0,04 & Rara & 50 & Acessória \\
\hline ACARI & 351 & 2,11 & Subdominante & 12,5 & Acidental \\
\hline ARACHNIDA & 251 & 1,51 & Recessiva & 100 & Constante \\
\hline ISOPODA & 5 & 0,03 & Rara & 25 & Acessória \\
\hline ISOPTERA & 2 & 0,01 & Rara & 12,5 & Acidental \\
\hline PULMONATA & 1 & 0,01 & Rara & 12,5 & Acidental \\
\hline TOTAL & 16658 & 100,00 & & & \\
\hline
\end{tabular}

Fonte: Dados da pesquisa (2015). 
Tabela 3 - Número de indivíduos (N), frequência (\%), constância e dominância (\%) de ordens coletadas com a armadilha Malaise 3 no período de fevereiro de 2014 a setembro de 2014.

\begin{tabular}{cccccc}
\hline \multicolumn{7}{c}{ Malaise 3 } \\
\hline ORDEM & $\mathbf{n}$ & Frequência (\%) & Classificação & Constância (C) & Classificação \\
\hline ARCHAEOGNATHA & 15 & 0,07 & Rara & 62,50 & Constante \\
BLATTARIA & 63 & 0,31 & Rara & 87,50 & Constante \\
COLEOPTERA & 468 & 2,30 & Subdominante & 100,00 & Constante \\
COLLEMBOLA & 1259 & 6,18 & Dominante & 100,00 & Constante \\
DERMAPTERA & 8 & 0,04 & Rara & 37,50 & Acessória \\
DIPTERA & 12722 & 62,50 & Eudominante & 100,00 & Constante \\
EMBIOPTERA & - & - & - & - & - \\
HEMIPTERA & 587 & 2,88 & Subdominante & 100,00 & Constante \\
HYMENOPTERA & 1391 & 6,83 & Dominante & 100,00 & Constante \\
LEPIDOPTERA & 1218 & 5,98 & Dominante & 100,00 & Constante \\
MANTODEA & 2 & 0,01 & Rara & 25,00 & Acessória \\
MECOPTERA & 1 & 0,005 & Rara & 12,50 & Acidental \\
MEGALOPTERA & 16 & 0,08 & Rara & 75,00 & Constante \\
NEUROPTERA & 21 & 0,10 & Rara & 62,50 & Constante \\
ODONATA & - & - & - & - & - \\
ORTHOPTERA & 10 & 0,05 & Rara & 50,00 & Acessória \\
PLECOPTERA & 1 & 0,005 & Rara & 12,50 & Acidental \\
PSOCOPTERA & 200 & 0,98 & Rara & 100,00 & Constante \\
STREPSIPTERA & 1 & 0,005 & Rara & 12,50 & Acidental \\
THYSANOPTERA & 6 & 0,03 & Rara & 62,50 & Constante \\
ACARI & 2191 & 10,76 & Eudominante & 37,50 & Acessória \\
ARACHNIDA & 175 & 0,86 & Rara & 100,00 & Constante \\
ISOPODA & - & - & - & - & - \\
ISOPTERA & - & - & - & - & - \\
PULMONATA & 1 & 0,005 & Rara & 12,50 & Acidental \\
\hline TOTAL & $\mathbf{2 0 3 5 6}$ & $\mathbf{1 0 0 , 0 0}$ & & & \\
\hline & & & &
\end{tabular}

Fonte: Dados da pesquisa (2015).

\section{DOMINÂNCIA ENTRE AS ORDENS REGISTRADAS}

As ordens categorizadas como raras compreenderam $57,9 \%$ das capturas para as armadilhas 1 e 2 e $66,6 \%$ para a armadilha 3 , o restante das ordens se enquadraram como dominantes (4 ordens) eudominantes ( 3 ordens), subdominantes ( 2 ordens) e recessiva (1 ordem) (Gráfico 6). 
Gráfico 6 - Percentual de dominância (D\%) entre as ordens coletadas nas três armadilhas.

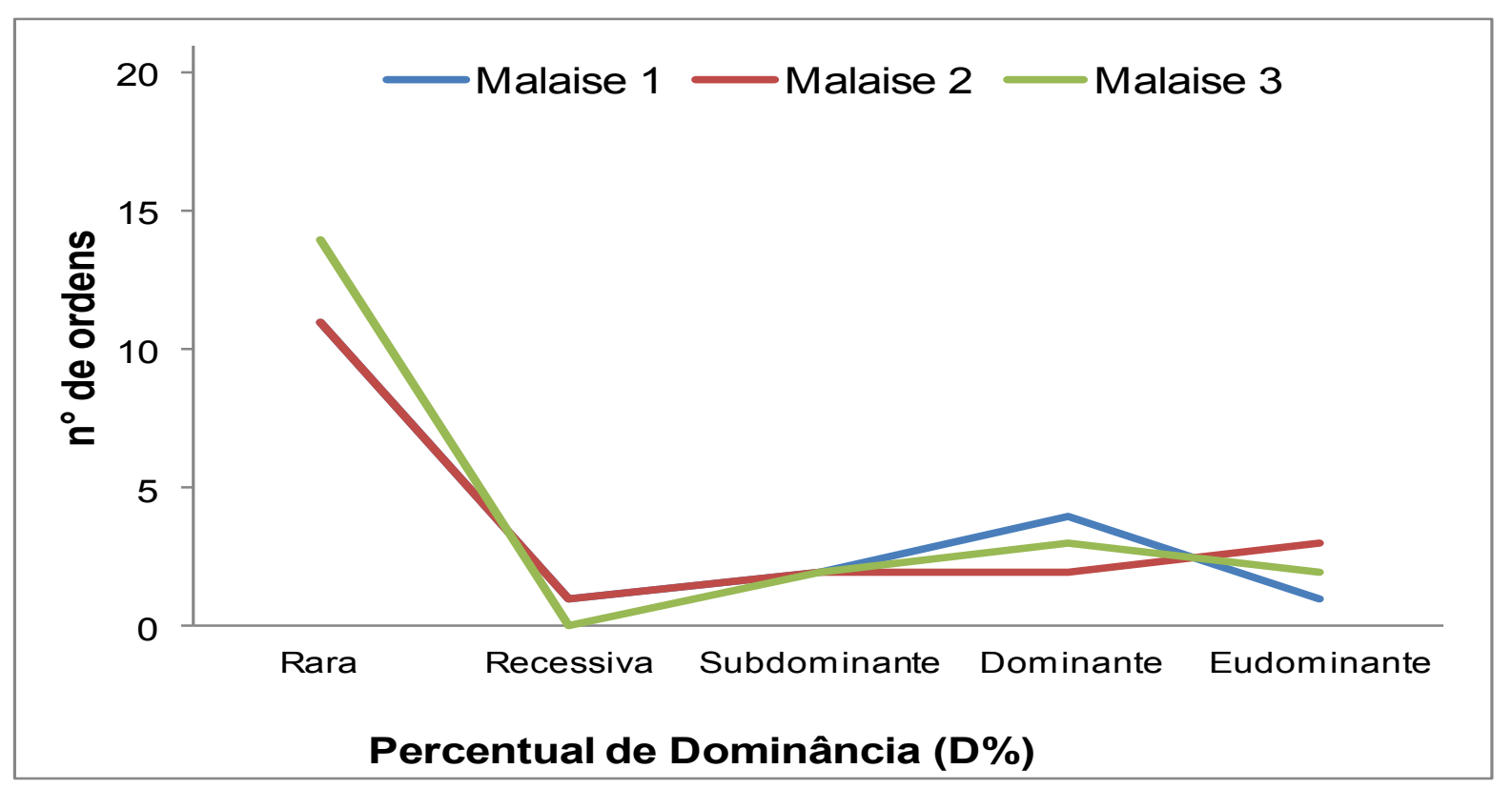

Fonte: Dados da pesquisa (2015).

\section{CONSIDERAÇÕES FINAIS}

Através dos dados obtidos, constatou-se que a entomofauna amostrada é composta por muitas ordens constantes e raras. Poucas ordens foram categorizadas como eudominantes e dominantes, no entanto, sua influência em termos de abundância foi superior a soma de todas as outras ordens coletadas.

Não houve grandes diferenças na composição faunística entre as três armadilhas, evidenciando um ambiente estável para a ocorrência das diversas ordens coletadas.

Já, para as estações do ano, o estudo demonstrou que o inverno apresentou o maior número de indivíduos coletados, resultado da influência dos picos de abundância de apenas três ordens.

A variedade de grupos de insetos encontrada pode indicar que o campus da Universidade do Contestado - Unidade de Marcílio Dias constitui um ambiente relevante para a conservação desse grupo. Os insetos representam importantes fontes de alimentos para muitos vertebrados, como anfíbios, répteis, aves e muitos mamíferos de pequeno porte. Daí sua importância ecológica e a necessidade de se conhecer um pouco mais os aspectos relacionados com a estrutura de suas comunidades. 


\section{REFERÊNCIAS}

BROWN, K.S.; FREITAS, A.V.L. Butterfly communities of urban forest fragments in Campinas, São Paulo, Brazil: structure, instability, environmental correlates, and conservation. Journal of Insect Conservation, New York, v. 6, n. 4, p. 217-231, 2002.

COLCEIRO, S.R.M. et al. Levantamento preliminar de insetos aquáticos de alguns municípios de Roraima, Brasil. Livro de resumos do $19^{\circ}$ Congresso Brasileiro de Entomologia: 16 a 21 de junho de 2002, Manaus, Amazonas: INPA, 320p. 2002.

COPATTI, C.E.; GASPARETTO, F.M. Diversidade de insetos em diferentes tipos de borda em um fragmento de Floresta Ombrófila Mista. Revista Biociências, Taubaté, v. 18, n. 2 , p. $32-40.2012$.

CUOZZO, M. et al. Variações na abundância de insetos capturados com armadilha malaise em região de cerrado ao sul de Minas Gerais. X Congresso de Ecologia do Brasil, 16 a 22 de Setembro de 2011, São Lourenço - MG. 2011.

DORNELES, L.L. Interações entre Euterpe edulis Mart. (Arecaceae) e insetos visitantes florais em sistema agroflorestal na Ilha de Santa Catarina. $111 \mathrm{p}$. Dissertação (Mestrado) - Universidade Federal de Santa Catarina, Florianópolis, SC, 2010.

DUTRA, R.R.C.; MARINONI, R.C. Insetos capturados com armadilha malaise na Ilha do Mel, Baía de Paranaguá, Paraná, Brasil. Revista Brasileira de Zoologia, v. 11, n. 21, p. 227 - 245, 1994.

GALLO, D. et al. Entomologia agrícola. Piracicaba: FEALQ, 2002.

GONÇALVES, P.A.S. Levantamento de Insetos Associados à Batata-Doce, Ipomoea batatas, com Uso de Armadilhas D’ágna, em Ituporanga, SC. Comunicação

Científica. Sociedade Entomológica Brasileira, v. 26, n. 1, p. 199-203, 1997.

HUSCH, P. E. et al. Entomofauna do entorno do reservatório de alagados, região dos campos gerais do Paraná, capturada através de armadilha malaise. Ciência, Biologia e Saúde, Ponta Grossa, v. 16, n. 1, p. 49-56, 2010.

JENNINGS, D.; HILBURN, D.J. Spiders (Araneae) captured in Malaise traps in spruce-fir forests of west-central Maine. Journal of Arachnology, v. 16, p. 85-94, 1998.

LARANJEIRO, A.J. Estabilidade da entomofauna num mosaico de plantação de eucalipto e áreas naturais de conservação. Tese (Doutorado) - Escola Superior de Agricultura "Luiz de Queiroz", Piracicaba, SP, 2003. 
MARCHIORI, C.H. et al. Parasitóides da subfamília Eucoilinae (Hymenoptera: Cynipoidea: Figitidae) coletados em um remanescente de mata de cerrado em Itumbiara, GO. Arquivos do Instituto Biológico, São Paulo, v. 68, n. 1, p. 65-67, 2001.

MARINONI, L.; BONATTO, S.R. Sazonalidade de três espécies de Syrphidae (Insecta, Diptera) capturadas com armadilha Malaise no Estado do Paraná, Brasil. Revista Brasileira de Zoologia, v. 19, n. 1, p. 95-104, 2002.

MARINONI, R.C.; DUTRA, R.R.C. Famílias de coleoptera capturadas com armadilha malaise em oito localidades do estado do Paraná, Brasil. diversidades alfa e beta. Revista Brasileira de Zoologia, v. 14, n. 3, p. 751 - 770, 1997.

OLIVEIRA, E.A. Coleópteros de uma ilha estuarina da Lagoa dos Patos, Rio Grande, Rio Grande do Sul, Brasil. 2006. Dissertação (Mestrado) - Universidade Federal do Paraná, Curitiba, 2006.

et al. Abundância e padrão sazonal da entomofauna de restinga em uma ilha do estuário da laguna Lagoa dos Patos, Rio Grande, RS, Brasil. Estudos de Biologia, v. 28, n. 64, p. 27-35, 2006.

OLIVEIRA, I.B.R. et al. Diversidade da entomofauna em uma área de Caatinga no município de Bom Jesus-PI, Brasil. Científica, Jaboticabal, v. 41, n. 2, p. 150-155, 2013.

PEREIRA, A. G. Uso de armadilhas malaise como estratégia de avaliação de bioindicadores em agrossistemas: diversidade e guildas de Braconidae em diferentes mosaicos vegetacionais da fazenda Chanchim (EMBRAPA), São Carlos, SP, Brasil. 2009. Dissertação (Mestrado) - UFSCar, São Carlos, 2009.

PERIN, C. Levantamento da diversidade de Pentatomoidea (HemipteraHeteroptera) e análise da influência do tamanho dos fragmentos florestais na riqueza de espécies no sul de Santa Catarina. 2009. Trabalho de Conclusão de Curso - Universidade do Extremo Sul Catarinense, UNESC, Criciúma, SC, 2009.

TOWNES, H. A light-weight Malaise trap. Entomological News, v. 83, p. 239-247. 1972.

VAS, J. et al. Study of Neuropteroidea (Raphidioptera, Neuroptera) communities by using malaise traps in an untreated orchard and its environment. Acta

Phytopathologica et Entomologica Hungarica, v. 36, n. 1-2, p.115-122, 2001.

WOLDAN, D.R.H. Análise faunística de Cerambycidae (Coleoptera) em duas situações florísticas no município de União da Vitória - Paraná. 2007.

Dissertação (Mestrado) - Universidade Comunitária Regional de Chapecó, 2007.

Artigo recebido em: 12/03/2015

Artigo aprovado em: 26/01/2016 